\title{
CAMBIOS EN EL PAISAJE INDUCIDOS POR DINÁMICAS SOCIOECONÓMICAS: UN ESTUDIO DE CASO CARTOGRÁFICO EN UNA MICROCUENCA DEL NORTE DEL TOLIMA (1955 A 2010)
}

\author{
Paola Andrea Rodríguez ${ }^{1}$ \\ Jairo Mora Delgado ${ }^{2}$ \\ Álvaro Briñez ${ }^{3}$
}

Recibido el 15 de octubre de 2014, aprobado el 6 de abril de 2015 y actualizado el 22 de octubre de 2015

DOI: 10.17151/luaz.2016.42.2

\section{RESUMEN}

El análisis cartográfico de una zona, permite identificar cambios del paisaje y de su dinámica antrópica en un territorio; estos reflejan procesos sociales generados por la interacción hombre-naturaleza. Se realizó una revisión histórica y cartográfica de los cambios del paisaje en una zona del municipio del Líbano (Tolima). Este trabajo se desarrollo en el marco del proyecto de investigación titulado "Agroecosistemas y cultura en fincas de la ecorregión cafetera del Tolima: evolución y conflicto" financiado por la Oficina Central de Investigaciones de la Universidad del Tolima. El objetivo del estudio fue documentar los cambios en los usos del suelo en el territorio de la microcuenca La Tigrera, especialmente la evolución de los agroecosistemas de café y su influencia en la transformación del paisaje. Se revisó información secundaria como mapas temáticos, literatura especializada e información institucional del Comité de Cafeteros del Tolima y de Cortolima. Por otra parte, se analizaron fotos aéreas (escala aproximada 1:60.000) de los años 1955, 1965, 1988 y 2010. Estas se procesaron de forma análoga a través de fotointerpretación, para cuantificar las áreas de ocupación de los usos del suelo presentes en el área de estudio. Se usó un ambiente SIG para crear cartografía temática de usos del suelo, utilizando programas especializados como AutoCAD 2010, Global Mapper, gvSIG 1.10 y ArcGis 1.10, con los cuales se procesaron imágenes tipo raster a partir de la digitalización de las fotos aéreas. Los resultados sugieren transformaciones del paisaje y patrones de uso de la tierra como consecuencia de las decisiones de actores que están inmersos en sistemas socioeconómicos y biofísicos, caracterizados por una compleja red de interrelaciones que evolucionan en el tiempo. Es notoria la reducción del área de bosques para dar paso primero a la caficultura y luego a las pasturas. Se concluye que las relaciones espaciales y de uso del suelo en la ecorregión cafetera del Tolima, son una expresión particular de las relaciones socioeconómicas originadas por la caficultura, lo que marca un ámbito tradicional en las decisiones que se toman en la familia, pero influenciadas por fuerzas macroeconómicas.

\section{PALABRAS CLAVES}

Usos del suelo, paisaje fragmentado, caficultura, medios de vida, pasturas.

\section{LANDSCAPE CHANGES INDUCED BY SOCIO-ECONOMIC DYNAMICS: MAPPING CASE STUDY IN A NORTHERN TOLIMA WATERSHED (1955 to 2010)}

\section{ABSTRACT}

The cartographic analysis of an area allows identification of the landscape changes and the anthropic dynamics in a territory; they reflect social processes generated by human-nature interaction. A historical and cartographic review of the landscape 
changes was carried out in an area of the Municipality of Líbano (Tolima). This work was developed within the framework of the research project "Agroecosystems and culture in farms of the coffee ecoregion of Tolima: evolution and conflict" financed by the Central Research Office at Universidad del Tolima. The aim of the study was to document changes in land use in the territory of La Tigrera watershed, especially the evolution of coffee agroecosystems and their influence on the transformation of the landscape. Secondary information was reviewed including thematic maps, specialized literature and institutional information of the Tolima Coffee Growers Committee and Cortolima. On the other hand, aerial photographs (approximate scale 1: 60,000) for the years 1955, 1965, 1988 and 2010 were analyzed and processed using analogue photo interpretation to quantify areas of occupation of land use present in the study area. A GIS environment was used to create thematic mapping of land use, using specialized programs like AutoCAD 2010, Global Mapper, gvSIG 1.10 and ArcGIS 1.10 with which raster images were processed from digitization of aerial photos. The results suggest changes in the landscape and patterns of land use as a result of the decisions of actors involved in socio-economic and biophysical systems, characterized by a complex network of relationships that evolve over time. The reduction of forest areas to give way to coffee first and then to pasture is notorious. It is concluded that the spatial relationships and land use in the Tolima coffee eco-region are a particular expression of the socio-economic relations caused by the coffee industry, marking a traditional area in the decisions made within the family but influenced by macroeconomic forces.

KEY WORDS: Land uses, fragmented landscape, coffee growth, livelihoods, pastures.

\section{INTRODUCCIÓN}

El paisaje cafetero ha sufrido muchos cambios a nivel histórico de carácter significativo, pero en Colombia pocos han sido los estudios que involucran a los componentes económicos, históricos y políticos el análisis ambiental. Se pueden mencionar tres autores que realizaron estudios de este tipo en el Líbano (Tolima), identificando modificaciones en el proceso de la tecnificación y las razones económico-políticas que impulsaban los cambios: Errazuriz (1986) y Ortiz (1989) (Guhl, 2004).

Para comprender el conflicto social que se ha generado a partir de la crisis de la economía cafetera en el Líbano (Tolima), es preciso analizar los cambios de uso del suelo y los procesos históricos en función de los choques generados en el entorno socioeconómico.

Esta investigación, es una aproximación al contexto en relación a la valoración cualitativa y cuantitativa de un sistema ambiental en conflicto social y natural. Para comprender la historia cafetera local, se debe tener en cuenta que hoy en día las poblaciones campesinas desempeñan diversas actividades en su portafolio productivo como alternativas a la situación de crisis en su principal actividad tradicional, en este caso la caficultura. Estas están relacionadas con el entorno y la cultura, pero también están mediadas por la necesidad del mercado, especialmente empujado desde dinámicas urbanas, en tanto se está en una sociedad globalizada mercantilizada (León, 2006).

Por lo anterior, se entiende que el cambio en el paisaje está íntimamente ligado a la forma como en cada momento histórico se realiza la apropiación de los recursos 
naturales en un determinado espacio por parte de una comunidad, lo que en última instancia se configura en un portafolio de actividades que inducen cambios en el paisaje por parte de las familias campesinas. Esta actividad humana ha sido la gran transformadora del paisaje en los últimos tres siglos (Aguayo et al., 2009), esto se ilustra con la pérdida de bosques y selvas tropicales en América Latina, para el año 2000 se habían reducido en más de un $50 \%$ de su cobertura original (Velázquez et al., 2002).

Generalmente, los choques entre el entorno natural y la dinámica socioeconómica, se expresan en las formas de enfrentar los cambios o conflictos de la vida cotidiana en función de los recursos naturales y sociales disponibles, los cuales son causas sociales y económicas de los cambios, en las escalas global, regional y local (Ojima et al., 1994; Skole et al., 1994). En este orden, un análisis multitemporal permite detectar cambios entre diferentes fechas de referencia, deduciendo la evolución del medio natural o las repercusiones de la acción humana sobre ese medio (Chuvieco, 1996). Estas relaciones se hacen visibles en la historia del uso del suelo en la zona del Líbano (Tolima). Así, los cambios en el paisaje son motivados por perturbaciones de orden natural como inundaciones, sequías, terremotos, entre otros, pero también estos cambios son originados por la actividad antrpica asociada a procesos económicos (Vega, 2005).

De esta manera, se entra a analizar el paisaje como una porción de la superficie terrestre con patrones de homogeneidad (Etter, 1991) que está formada por un grupo de sistemas asociados a la actividad natural y humana. En este sentido, es necesario caracterizar los cambios de paisaje relacionados con los diferentes usos que se le han dado al suelo en la zona de estudio, sin dejar de lado la población que reside en esas tierras, ya que la relación hombre-ambiente es una relación culturalmente establecida y mediada por la tradición.

Las coberturas vegetales y las formas naturales como producto de la interacción espacio-tiempo, denotan cambios en las formaciones económicas, es decir, estos cambios son motivados por la necesidad del hombre de adaptarse al ambiente modificándolo, por lo tanto aparece el análisis cartográfico que constituye una especie de radiografía de los usos del suelo, que se manifiestan "como el resultado de la asociación de elementos biológicos vegetales característicos, los cuales conforman unidades estructurales y funcionales" (Etter,1991,16).

Se comparte la idea de que la historia ambiental se presenta como un campo importante de análisis geográfico, en tanto los paisajes sintetizan la relación naturaleza-sociedad, es decir, si se comprende cómo se ha transformado un paisaje, se pueden llegar a identificar qué prácticas son sostenibles y amigables con el entono (Guhl, 2004).

La evaluación del cambio de cobertura y uso del suelo, en términos espaciales, es un recurso importante a nivel de planificación de cuencas en Colombia, pero infortunadamente no se ha dado el espacio al contexto histórico, el cual involucra elementos temporales y temáticos que podrían indicar conflictos locales. Esto se debe, en la mayoría de los casos, a la inexistencia de una base de datos especializada y a restricciones de carácter presupuestal en las entidades encargadas de la gestión ambiental (Pérez y Bosque, 2008), lo cual indica que los tomadores de decisión están alejados de lo que pasa en la realidad, y los procesos de ordenamiento al no tener en cuenta esta variable, desconocen el contexto, lo que implica una gestión ambiental sesgada.

Los objetivos de este artículo son: Caracterizar histórica y geográficamente el cambio del paisaje en el municipio del Líbano (Tolima), y modelar las diferentes variaciones del paisaje de la zona de estudio desde 1955 hasta 2010, con la 
elaboración de mapas históricos de la evolución y cambio de paisaje en la zona de estudio.

\section{METODOLOGÍA}

Zona de estudio

El estudio se realizó en la zona rural del municipio del Líbano $\left(04^{\circ} 53,760^{\prime} \mathrm{N}\right.$ $75^{\circ} 05,154^{\prime}$ O), con una elevación máxima de 2000 y una elevación mínima de 1400 msnm dentro de la microcuenca La Tigrera constituyente de la cuenca del río Recio, con una extensión aproximada de 4446 ha. Administrativamente esta localidad es conocida como La Tigrera. (Figura 1).

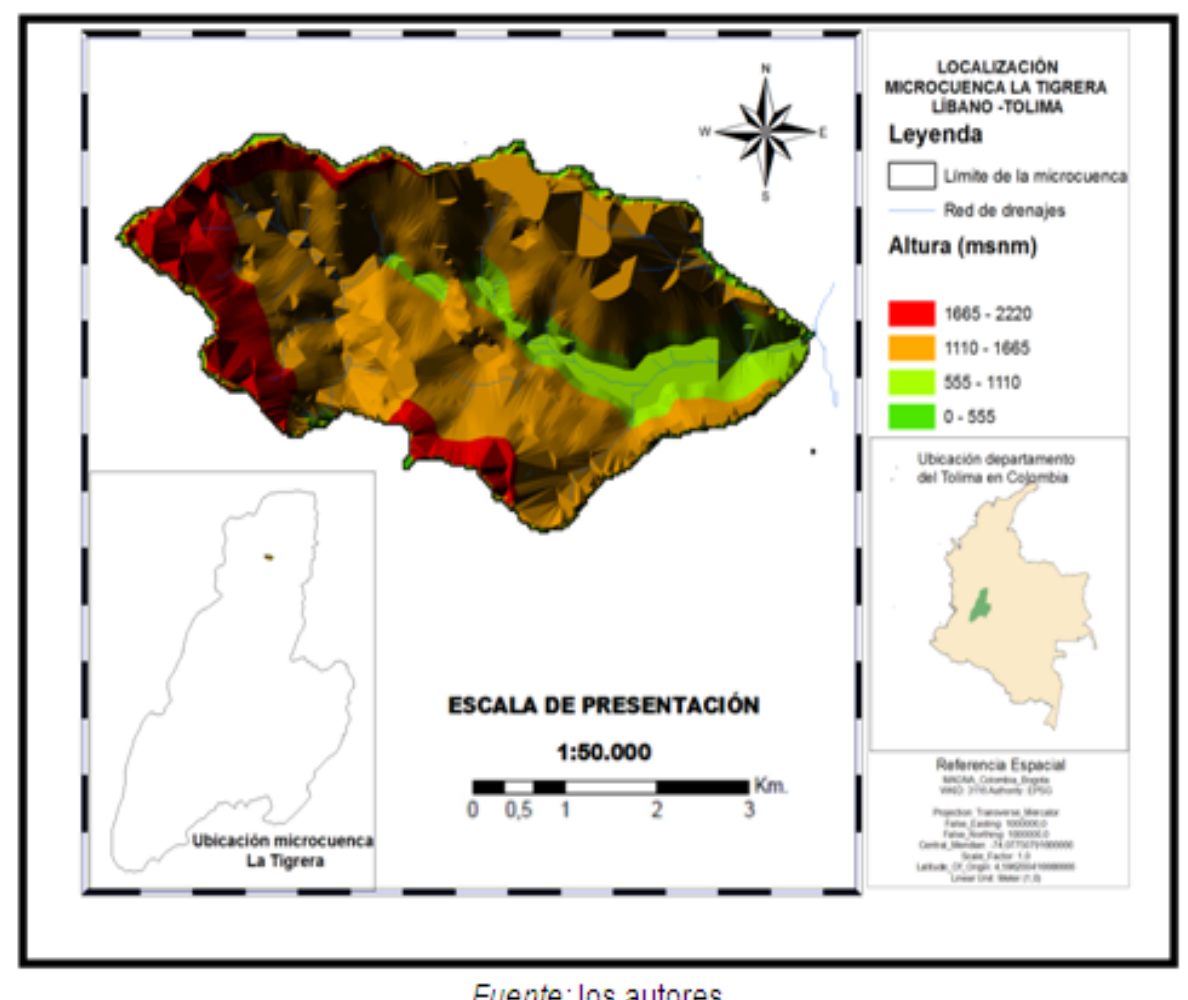

Figura 1. Ubicación espacial de la zona de estudio.

Obtención de información

- Análisis histórico del cultivo del café: Se revisó información pre- elaborada del objeto de estudio como mapas temáticos, literatura especializada e información institucional del Comité de Cafeteros del Tolima y de Cortolima.

- Trabajo en ambiente SIG para producción mapas históricos: En las fotografías aéreas (Tabla 1) se definieron las categorías de tipo de cobertura. El proceso de ortorrectificación se realizó con el programa E-Foto, después se escalaron todas las imágenes en el programa AutoCAD 2010, tomando como referencia la escala 1:60.000; además, se digitalizaron los polígonos de las diferentes coberturas presentes en los cuatro periodos de tiempo, y se sacaron curvas a nivel con ayuda del programa Global Mapper 15; el programa Arc-GIS 10.1 se empleó para 
delimitar la microcuenca La Tigrera y realizar un modelado en 3D. Complementariamente, se hizo un recorrido por la zona de estudio, tomando puntos GPS para la posterior georreferenciación.

En este sentido, se aplicó el método de análisis geográfico desde la perspectiva del estudio de los cambios en el uso del suelo en un territorio, especialmente la evolución del monocultivo del café y su influencia en la transformación del paisaje. Se realizó una verificación en campo para la identificación de coberturas de suelo y la corroboración en las imágenes satelitales.

\section{Cuantificación de los cambios}

El cambio de uso de suelo fue cuantificado usando la ecuación propuesta por la FAO (1996), para calcular la tasa de cambio anual (TCA):

$\mathrm{TCA}=[\mathrm{S} 2 / \mathrm{S} 1] 1 / \mathrm{n}-1$

Donde: TCA: tasa de cambio anual, $\mathrm{S} 2=$ área en fecha 2, S1 = área en fecha 1, $\mathrm{n}$ = número de años entre las dos fechas.

Ese análisis cartográfico permitió analizar los cambios en el uso del suelo, los cuales fueron cotejados con eventos sociopolíticos y económicos relacionados que posiblemente influyeron en la reconfiguración del paisaje.

Tabla 1. Líneas de vuelo de las fotografías aéreas analizadas

\begin{tabular}{lcc}
\hline Número de vuelo & Fotos & Año \\
\hline M-40 & 3655,3656 & 1955 \\
R-749 & 10,11 & 1965 \\
C-2397 & 216,217 & 1988 \\
C-2684 & 227,228 & 2010 \\
\hline
\end{tabular}

Fuente: Ios autores.

\section{RESULTADOS Y DISCUSIÓN}

La zona de estudio es caracterizada históricamente por la coexistencia de grandes haciendas y muchas parcelaciones, en las cuales las familias campesinas están sujetas por sus condiciones de vulnerabilidad al conflicto social, a causa de los cambios en la economía del café, las presiones de la violencia, las enfermedades y plagas del cultivo, lo que en última instancia condiciona la forma de producción cafetera en esta zona del Tolima. En la década del veinte al treinta, el Líbano se proyecta como un atractivo centro cafetero para inversionistas nacionales y extranjeros, particularmente alemanes y norteamericanos, quienes vincularon sus capitales a la producción, beneficio y comercialización del café (Andrade, 2012),

La mayor participación en la producción local estaba en manos de las grandes haciendas. En 1932 el censo cafetero menciona 7 plantaciones con más de 100.000 cafetos que generaban el $45,8 \%$ del total de la producción, mientras que 136 fincas restantes solo participaban con el $15,6 \%$ del total del café producido (Rincón, 2001) 
El abandono del campo (1955)

Se observa en la Tabla 2 la extensión del área sembrada en café, la variedad arábigo Typica en proporción para 1955 es de solo 3,2\% de la superficie de la microcuenca, posiblemente porque desde mediados de los años cincuenta, miles de haciendas estaban llamadas a renovar sus plantaciones, debido a su bajo nivel productivo. No obstante, tal parece que la renovación de los cafetales "no era una buena acción económica por la época de la violencia" (Ramírez, 2009, 168), en consecuencia el paisaje retornó al rastrojo, por lo cual en pocos años la regeneración natural y los bosques secundarios volvieron a ser el uso del suelo más extendido en esta década $(60,6 \%)$ seguida de las pasturas para ganadería extensiva $(5,7 \%)$. Los cultivos de café tradicional, con las variedades de la especie Coffea arabica, se tornaron viejos e improductivos. Ver figura 2

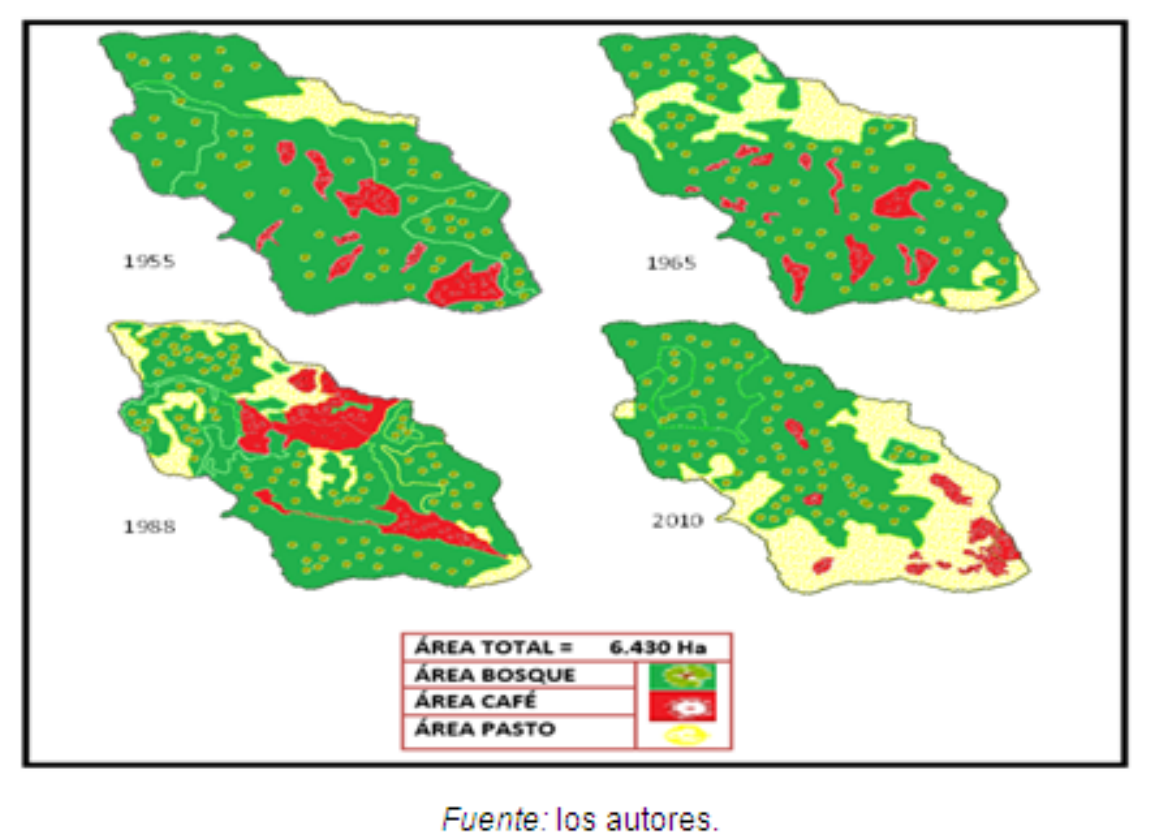

Figura 2. Uso del suelo en la zona de estudio en cuatro décadas, en la microcuenca La Tigrera (Líbano, Tolima).

El área de regeneración natural representa la superficie de mayor proporción; posiblemente debido al abandono del campo por la violencia y sumado a la falta de tecnología en el cultivo del café, permitieron la regeneración del bosque. De hecho, en este periodo se recrudeció el malestar campesino especialmente en las zonas rurales del Tolima, invadiendo propiedades y disolviendo el régimen de haciendas que predominaba, lo cual afectó las inversiones de capital en esta zona y fortaleció la pequeña unidad productiva en torno al grupo familiar. La década de 1950 constituyó una época en la que se diezmó la población del Líbano e hizo que la mayoría de habitantes rurales abandonaran los campos para sobrevivir, trayendo como consecuencia la emigración hacia los centros urbanos más cercanos (Andrade, 2012).

La violencia se circunscribía de manera particular a los corregimientos de San Fernando, Santa Teresa y Tierradentro. Es decir, el sector sur del municipio, lugar de alta densidad poblacional y de concentración de las grandes haciendas y de los más prósperos centros de producción local de café (Rincón, 2001). 
Los albores de la revolución verde (1965)

La época de la violencia determina varios elementos constitutivos para el desarrollo de la caficultura tecnificada. A raíz del conflicto bélico muchos terratenientes de la zona fueron desplazados o muertos, esto propició la fragmentación de las grandes haciendas, lo cual fue apoyado por el movimiento de reforma agraria de los aparceros o arrendatarios en Líbano, llegando a generar la modernización de las relaciones laborales, y generando la parcelación de varias de ellas, como La Aurora y La Trinidad (Rodríguez, 2013).

En 1965 aumenta el área de café a 4,5\% al igual que las pasturas que se incrementan notoriamente $(11,2 \%)$, respecto a la década anterior, mientras el área de regeneración natural disminuye $(53,7 \%)$. Situación que implicó cambios cruciales en los sistemas agropecuarios de la zona, con la maximización del uso de la tierra con el monocultivo del café tecnificado, que genera una nueva forma de producción del café, renacida bajo la egida de la revolución verde y la variedad de café de la Federación Nacional de Cafeteros. Implicando la maximización del uso de la tierra y la fuerza laboral del grupo familiar en torno a la producción del grano.

A partir de 1960, los investigadores de Cenicafé, desarrollaron las tecnologías que fueron fundamentales para el impresionante progreso del cultivo en la segunda parte del siglo XX. Los elementos fundamentales fueron los estudios con la variedad Caturra, de porte bajo, alta productividad, buena calidad en taza, y excelente adaptación a las condiciones ecológicas de los Andes (Cadena, 2005).

A partir de 1963, se inició la siembra de la variedad Caturra, lo que implicó la pérdida de rastrojo y plantas de sombrío, dibujando un paisaje más exiguo basado en la ampliación del monocultivo cafetero. Las pasturas comienzan a posesionarse en el paisaje cada vez con más fuerza, como resultado de la roza ${ }^{4}$, para ampliar terreno de cultivo, tal como se evidencia en la Tabla 2, en la tasa de cambio (TAC) de la Figura 3. Posiblemente, la alta TAC expresada en la pasturas sea el resultado de la apertura de nuevas tierras en bosques alto-andinos, pero también el abandono del campo de los hacendatarios por cuenta de la violencia partidista de la época.

Tabla 2. Cobertura del suelo y tasa de cambio (TAC) en el período 1955-2010, en la microcuenca La Tigrera (Tolima, Colombia)

\begin{tabular}{lcccccccc}
\hline & \multicolumn{2}{c}{1955} & \multicolumn{2}{c}{1965} & \multicolumn{2}{c}{1988} & \multicolumn{2}{c}{2010} \\
\cline { 2 - 9 } Usos & ha & $\%$ & ha & $\%$ & ha & $\%$ & ha & $\%$ \\
\hline Bosque & 3879,6 & 60,6 & 3440,9 & 53,7 & 2961,6 & 46,0 & 2693,8 & 41,7 \\
Café & 204,2 & 3,2 & 285,8 & 4,5 & 695,9 & 10,8 & 204,8 & 3,2 \\
Pasto & 362,3 & 5,7 & 719,4 & 11,2 & 788,6 & 12,3 & 1547,5 & 24,0 \\
Total & 4446,1 & 100,0 & 4446,1 & 100,0 & 4446,1 & 100,0 & 4446,1 & 100,0 \\
\hline
\end{tabular}

Fuente: los autores.

Bonanza cafetera, año 1988

La alta TAC calculada para el periodo $1965-1988$ es el reflejo de la época de bonanza cafetera (Tabla 2). El sistema de producción cafetero a partir del año setenta, hace que el campesino se especialice en el monocultivo del café, dejando 
de un lado la diversidad en sus parcelas y creando dependencia de la comercialización del grano, esto con los años constituyó su vulnerabilidad a las fluctuaciones del comercio internacional. Esto fue exacerbado por los servicios de extensión de la FNC, puesto que a partir de 1975 se generaliza la asistencia técnica por parte de la Federación en el ámbito rural, mediante la conformación de Grupos de Amistad, sujetos de capacitación necesarios para el establecimiento y manejo de la plantación (Rodríguez, 2013).

El mapa del año 1988 identifica que el área de café ya se había incrementado a un $10,8 \%$ de la superficie de la microcuenca, como resultado de una TAC significativa de un $4 \%$ anual en los anteriores 23 años. Es la época en la cual se generaliza la siembra de la variedad Colombia que no necesita sombrío, sin embargo también es evidente un aumento de pasturas $(12,3 \%)$ para ampliación de la frontera agrícola, aunque con menos TAC que el periodo anterior (0,04\%).

Este período implica, igualmente, la intensificación de los procesos de renovación de cafetales y el establecimiento de la nueva variedad Colombia (Rincón, 2001). Las condiciones ambientales de la zona de estudio se alteraron de forma significativa, ya que la producción de café sin sombrío indicaba una transformación del paisaje a expensas de aumentar la producción del cultivo, creado nuevas zona de agricultura y limitando la cobertura natural de la zona.

La producción de café sin sombra, modifica de manera sustancial el paisaje nativo.

El Caturra no necesitaba de la protección de los grandes árboles, por lo que empezaron a ser destruidos, disminuyendo la fauna y flora naturales. Tampoco eran necesarias las mejoras de plátano, maíz y otras de autoconsumo doméstico, así como la diversidad ganadera de las fincas. De esta manera, disminuyó el índice de aguas naturales y de maderas (Ramírez, 2009).

Los resultados de esta nueva práctica, dieron lugar a un una zona dedicada solamente a la producción de café y los rastrojos como el café asociado con plátano disminuyeron. La supresión del sombrío generó la eliminación del sotobosque, aumentando el grado de erosión, la pérdida de biodiversidad, la falta del control biológico natural, facilitando la aparición de plagas y enfermedades como la Roya y la Broca (Vega, 2005).

En este orden de ideas, a finales de la década de los ochenta, el paisaje sufrió variaciones en el número de plantas de café, porque algunos campesinos tuvieron que quemar sus cultivos para evitar la diseminación de los problemas fitosanitarios, además se agudizó la contaminación por químicos; pero a pesar de lo anterior, el uso del suelo siguió destinado a la siembra de café sin sombra, el área de regeneración natural siguió restringida y los cultivos diferentes se mantuvieron al margen de la producción total de la zona.

\section{Época de la crisis por finalización del pacto de cuotas}

Las tendencias ilustradas en la Tabla 2 y la Figura 2 constituyen evidencias del nuevo escenario del uso del suelo después de los años noventa, entre tanto los caficultores tuvieron que entrar a diversificar aumentando la potrerización de zonas de laderas, lo cual tiene un impacto en los procesos de erosión y la pérdida de diversidad de la zona; por una parte, el área de bosques continúa en descenso, aunque con menor tasa que en los periodos anteriores, y una nueva situación nunca antes vista se vislumbra en la tendencia de áreas destinadas a café. De hecho, en este periodo es notoria la TAC negativa de las áreas dedicadas a café y la aceleración en la TAC de las pasturas, lo que denota la crisis que comienza para los productores de café ante la finalización del pacto de cuotas en 1988. 
La respuesta del Estado fue promover los "alivios económicos" una categoría relativa y polémica, situación que prolongó la agonía del caficultor, al postergar el tiempo de la deuda, posponiendo las consecuencias sin erradicarlas (Robledo, 1998), en tanto que se mantenían las condiciones de baja producción debido a la Broca y en menor proporción a la Roya, además de la difícil comercialización del grano.

Un número considerable de campesinos perdieron sus propiedades y se vieron enfrentados a la necesidad de recurrir a otras fuentes de ingresos, bien trabajando como asalariados rurales o desplazándose a los centros urbanos en busca de ofertas de trabajo. En términos de Rincón (2001), indica que el desplazamiento ha propiciado el fortalecimiento de sectores subnormales en los cascos urbanos de aquellas poblaciones dependientes de la economía agrícola, en las que la crisis productiva ha redundado directamente en su inestabilidad social y económica: la proliferación de asentamientos "subnormales" y el fortalecimiento de actividades económicas como el subempleo.

Al final de la década del noventa, el plano social era muy conflictivo en el sector rural del Líbano. Al ser el único producto de comercialización en la zona y tener bajas en su precio creó problemas en la canasta familiar, ya que el café dejó de ser objeto de ingreso para las familias campesinas, y de otra parte, al no tener otras opciones de cultivos en sus predios los campesinos llegaron a la crisis, por problemas de abastecimiento, fenómeno que ocasionó procesos migratorios del campo a los cascos urbanos más cercanos, desplazándose así gran parte de la población activa a nivel laboral.

Se observa en el mapa (Figura 2) que la zona de café disminuyó en comparación con la época de la bonanza, pasó de 10,8\% en 1988 a 3,2\% en 2010; la regeneración natural representa el $41 \%$ y las pasturas el $24 \%$. Es de resaltar que la TAC demuestra un incremento significativo del uso del suelo en pasturas, aunque para el desarrollo de una ganadería extensiva (Figura 3). Hay una ligera pérdida del área en bosques respecto al periodo anterior.

Rodríguez (2013) demuestra que el paisaje cafetalero en los últimos 15 años tendió a diversificarse, con la incursión de otros productos agrícolas y pecuarios como estrategia para incrementar los medios de vida de la familia campesina de la zona cafetera. 


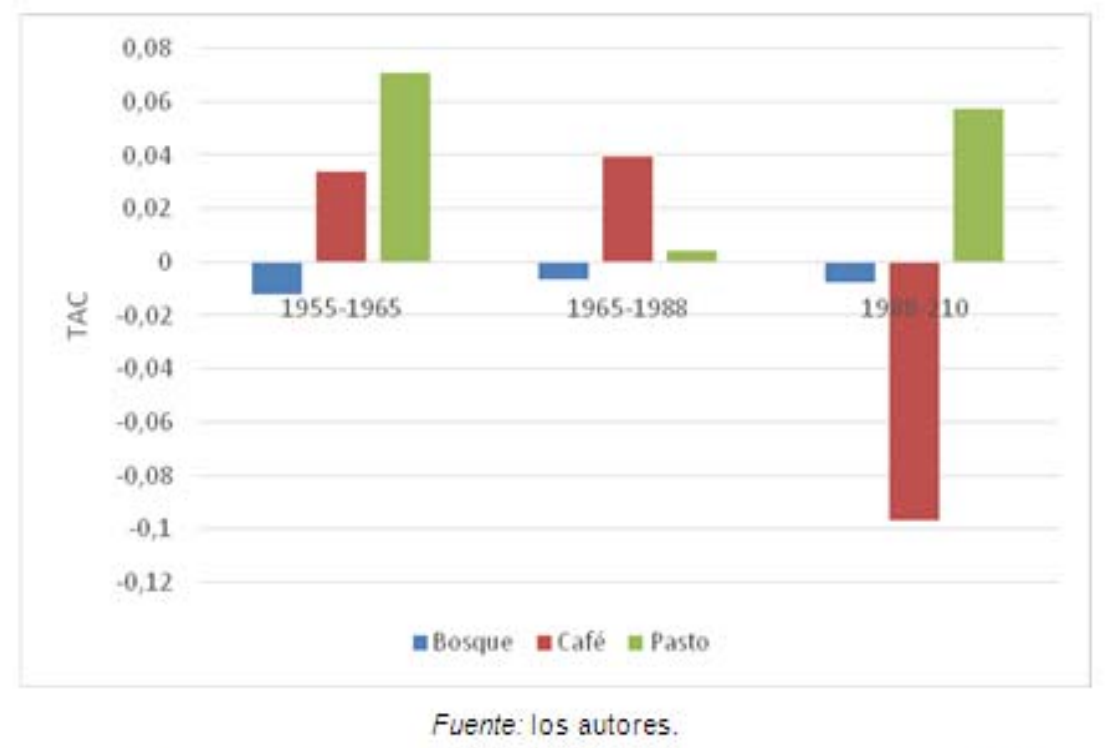

Figura 3. Tasa anual de cambio de uso de suelo en la microcuenca La Tigrera,

cuenca del río Recio ( 4446,1 ha).

\section{CONCLUSIONES}

Se puede argumentar que el problema de la crisis del café, no solo está asociado al productor directo, sino además motivado y determinado por la política económica del país, que muestra una vocación extractivista desde el periodo de la Colonia, lo que determina una herencia de dominación frente al mercado internacional, determinándose procesos de pobreza y pérdida de la diversidad natural por la extracción de recursos. Además de la pérdida de la identidad cultural en la medida de que no se genera una relación ambiental adecuada entre el campesino y su finca.

Es evidente que la dinámica en la economía cafetera y en especial la crisis de este sector, ha sido la mayor causa de cambios en el paisaje al menos en los últimos 40 años. De hecho, la reducción en el área sembrada de café es un indicador de la crisis del sector, lo que ha implicado disminución de empleos y merma en los ingresos. El uso del suelo que se ha incrementado de manera significativa es el paisaje de pasturas, lo cual constituye un indicador de conflictos ambientales dada la topografía de ladera en donde la ganadería extensiva puede ser un motor de problemas ambientales y conflictos sociales.

Se sugiere que el uso de estudios relacionales del contexto con la representación geográfica en mapas temáticos es una herramienta importante para la planificación de zonas estratégicas, a escala de microcuencas y cuencas. Se hace necesario desarrollar mapas temáticos a nivel histórico para poder hacer una adecuada lectura del paisaje y así generar insumos base para la toma de decisiones de tipo administrativo, en las cuales se tenga en cuenta el contexto ambiental y cultural de la zona de estudio 


\section{REFERENCIAS}

- Aguayo, A., Pauchard, A., Azócar, G. y Parra, O. (2009). Cambio del uso del suelo en el centro sur de Chile a fines del siglo XX. Entendiendo la dinámica espacial y temporal del paisaje. Revista Chilena de Historia Natural, 82, 361-374.

- Andrade, S. (2012). Impactos del conflicto político militar en la vida cotidiana del municipio del Líbano Tolima entre 1991 y 2007. Tesis de Maestría.Universidad del Tolima, Ibagué.

- Cadena, G. (2005). Desarrollos Científicos de Cenicafé. Revista Academia Colombiana Ciencia, XXIX (110), 89-99.

- CHUVIECO, E. (1996): Fundamentos de Teledetección espacial. Madrid: Editorial Rialp

- Errazuriz, M. (1986). Cafeteros y cafetales del Líbano: cambio tecnológico y diferenciación social en una zona cafetera. Bogotá: Universidad Nacional de Colombia.

- Etter, A. (1991). Introduccion a la ecología del paisaje: un marco de integracion para los levantamientos rurales. Bogotá, D.C.: IGAC.

- FAO. (1996). Survey of tropical forest cover and study of change processes. Roma,130.

- Guhl, A. (2004). Café y cambio de paisaje en la zona cafetera colombiana entre 1970 y 1997. Cenicafé, 55, 29-44.

- León, J. (2006). Estrategias de vida en familias cafeteras y su relación con la riqueza etnobotánica de fincas en el departamento de Caldas, Colombia. San José: CATIE.

- Ojima, D., Galvin, K., Turner, II. The global impact of land-use change. Bioscience. Vol 44(5): 300-304

- Ortiz, A. (1989). Sombríos y caturrales del Líbano-Tolima, transformación y crisis ecológica de un paisaje cafetero. Bogotá: IGAC.

- Pérez, U. y Bosque, J. (2008). Transiciones de la cobertura y uso de la tierra en el periodo 1991-2005 en la Cuenca del Río Combeima, Colombia. Serie Geográfica - Profesora María de los Ángeles Díaz Muñoz, In Memoriam, 163-178.

- Ramírez, R. (2009). La broca del café en Líbano. Impacto socioproductivo y cultural en los años 90. Revista de Estudios Sociales, 32, 158-171.

- Rincón, J. (2001). De Café a oscuro: conflicto social y producción cafetera en el norte del Tolima. Bogotá: Facultad de Ciencias Humanas, Universidad Nacional de Colombia.

- Robledo, J. E. (1998). El café en Colombia. Bogotá: Áncora.

- Rodríguez, P. (2013). Agroecosistemas y Cultura en fincas de la Ecorregión Cafetera del Tolima: evolución y conflicto. Tesis M.-Sc, Universidad del Tolima. Ibagué, Colombia. 
- Skole, D., Chomentowski, W., Salas, W. y Nobre, A. (1994). Physical and Human Dimensions of Deforestation in Amazonia. Bioscience, 44(5), 314-322.

- Vega, A. (2005). Estudio multi-temporal de cambio de coberturas en un sector de los municipios de Circasia y Filandia (Quindío, Colombia). Una aproximación desde los Modelos Basados en Agentes (ABM). Bogotá, D.C.: Pontificia Universidad Javeriana, Facultad de Estudios Ambientales y Rurales, Carrera de Ecología.

- Velázquez, A., Mas, J., Díaz , J., Mayorga, R., Alcántara, P., Castro, R.,Fernández, T.,Bocco, G.,Ezcurra, E.,Palacio, J.,(2002). Patrones y tasas de cambio de uso del suelo en México. Gaceta Ecológica, 62,21-37

1. Est. PhD en planificación y gestión integral de cuencas hidrográficas, Profesora Asistente-catedrática, Grupo de Investigación Centro de Estudios Rurales (CERES), Universidad del Tolima. Ibagué, Colombia. paorodriguezr@ut.educo

2. Ph.D. En Sistemas de Producción Agrícola Tropical Sostenible, Profesor Asociado, Grupo de Investigación Sistemas Agroforestales Pecuarios, Universidad del Tolima. Ibagué, Colombia. jrmora@ut.edu.co

3. Estudiante de IX semestre de Ingeniería Forestal, Grupo de Investigación Centro de Estudios Rurales (CERES), Universidad del Tolima. Ibagué, Colombia. abrinezs@ut.edu

4. La técnica "roza, tumba y quema" es una técnica que ha sido muy empleada por los agricultores, para poder practicar el cultivo y extender sus tierras.

5. Los alivios económicos fueron una política de emergencia direccionados desde el Gobierno central en los años de 1991 a 2000.

Para citar este artículo: Rodríguez, P. A., Mora Delgado, J. y Briñez, Á. (2016). Cambios en el paisaje inducidos por dinámicas socioeconómicas: un estudio de caso cartográfico en una microcuenca del norte del Tolima (1955 a 2010). Revista Luna Azul, 42, 3-14. Recuperado de http://200.21.104.25/lunazul/index.php?option=com_content\&view=article\&id=124 